\title{
A RESISTÊNCIA AOS ANTIMONIAIS PODE LEVAR AO AUMENTO DA VIRULÊNCIA EM LEISHMANIA SPP.?
}

\author{
CAN RESISTANCE TO ANTIMONIALS LEAD TO INCREASED \\ VIRULENCE IN LEISHMANIA SPP.?
}

\author{
Laísa Vilar Cordeiro ${ }^{1}$ \\ Pedro Thiago Ramalho de Figueiredo ${ }^{2}$ \\ Thamara Rodrigues de $\mathrm{Melo}^{3}$ \\ Giulian César da Silva Sá ${ }^{4}$ \\ Aleson Pereira de Sousa ${ }^{5}$
}

\begin{abstract}
RESUMO: Leishmanioses são doenças negligenciadas, que acometem principalmente países tropicais e populações de baixa condição socioeconômica. $\mathrm{Na}$ prática clínica, a principal ferramenta para o combate da leishmaniose ainda é a farmacoterapia. Contudo, o arsenal terapêutico é bastante limitado. Na maioria dos países, como no Brasil, as drogas de primeira escolha para o tratamento das infecções por Leishmania sp. são os antimoniais e o tratamento possui várias limitações, dentre elas o crescente surgimento de parasitos resistentes a estas drogas. Estudos em procariotos e eucariotos demonstram que a resistência a drogas está relacionada a alterações no fitness dos micro-organismos, onde é possível observar modificações no crescimento, virulência e transmissibilidade entre hospedeiros. Visto que tais alterações podem influenciar diretamente no curso da infecção, alterando o prognóstico clínico, é relevante que essa associação entre resistência a drogas e virulência/fitness parasitário seja melhor estudada. Esta revisão bibliográfica objetivou avaliar se a resistência aos antimoniais está correlacionada com o aumento da virulência de parasitos do gênero Leishmania. Trata-se de um estudo descritivo, realizado a partir das pesquisas bibliográficas e documentais para subsidiar a discussão do tema. A pesquisa foi realizada entre os meses de dezembro a fevereiro de 2020. Estudos apontam que parasitos resistentes aos antimoniais apresentam maiores parâmetros de virulência in vitro e in vivo. Tais estudos ainda são escassamente encontrados na literatura, mas apontam a

\footnotetext{
${ }_{1}^{1}$ Doutorandos em Produtos Naturais e Sintéticos Bioativos, Universidade Federal da Paraíba (UFPB);

${ }^{2}$ Doutorandos em Produtos Naturais e Sintéticos Bioativos, Universidade Federal da Paraíba (UFPB);

${ }^{3}$ Doutorandos em Produtos Naturais e Sintéticos Bioativos, Universidade Federal da Paraíba (UFPB);

${ }^{4}$ Doutorando em Bioquímica, Universidade Federal do Rio Grande do Norte (UFRN);

${ }^{5}$ Doutorando em Desenvolvimento e Inovação Tecnológica em Medicamentos, Universidade Federal da Paraíba (UFPB).
} 
necessidade dessa correlação ser melhor estudada, de modo a esclarecer as alterações moleculares envolvidas nesses processos e poder otimizar a farmacoterapia dessas infecções, impactando diretamente na prática clínica.

Palavras chave: Leishmania spp.; leishmaniose; resistência; virulência.

ABSTRACT: Leishmaniasis are neglected diseases, which mainly affect tropical countries and populations with low socioeconomic status. In clinical practice, the main tool for combating leishmaniasis is the pharmacotherapy. However, the therapeutic arsenal is quite limited. In most countries, as in Brazil, the first choice drugs for the treatment of Leishmania sp. are antimonials and the treatment has several limitations, among them the growing appearance of parasites resistant to these drugs. Studies in prokaryotes and eukaryotes demonstrate that drug resistance is related to changes in the fitness of microorganisms, where it is possible to observe changes in growth, virulence and transmissibility between hosts. Since these changes can directly influence the course of the infection, changing the clinical prognosis, it is relevant that this association between drug resistance and parasitic virulence/fitness is better studied. This bibliographic review aimed to assess whether resistance to antimonials is correlated with increased virulence in Leishmania parasites. This is a descriptive study, based on bibliographic and documentary research to support the discussion of the theme. The survey was conducted between December and February 2020. Studies show that parasites resistant to antimonials have higher virulence parameters in vitro and in vivo. Such studies are still rarely found in the literature, but they point out the need for this correlation to be better studied, in order to clarify the molecular changes involved in these processes and to be able to optimize the pharmacotherapy of these infections, directly impacting clinical practice.

Key-words: Leishmania spp.; leishmaniasis; resistance; virulence. 


\section{INTRODUÇÃO}

Leishmanioses é o nome dado ao grupo de doenças não contagiosas, infecciosas, causadas por protozoários do gênero Leishmania e que são transmitidas ao homem pela picada de flebotomíneos fêmeas infectadas. Estão inseridas no grupo das doenças negligenciadas, que acometem principalmente países tropicais e populações de baixa condição socioeconômica, estando associada à desnutrição, moradia precária, deslocamento da população, alterações climáticas e ambientais (MURRAY et al., 2005; FEASEY et al., 2010).

A desnutrição, principalmente estando relacionada a uma dieta pobre em proteínas, ferro, vitamina A e zinco aumentam o risco da infecção progredir para formas mais agressivas, visto que essas deficiências nutricionais ocasionam comprometimento do sistema imunológico, de diferentes maneiras. Condições precárias de moradia e aspectos sanitários domésticos inadequados como falta de manejo de resíduos ou esgoto a céu aberto podem aumentar os locais de reprodução e descanso de flebotomíneos, bem como o acesso a humanos. Os mosquitos transmissores da doença são atraídos também por moradias lotadas, pois este ambiente torna- se uma abundante fonte de sangue para seu repasto. Comportamentos humanos, como dormir ao ar livre ou no chão, também podem aumentar o risco de adquirir leishmanioses (WHO, 2020).

Epidemias de leishmaniose são frequentemente associadas à migração e ao movimento de pessoas não imunes para áreas com ciclos de transmissão existentes. A exposição ocupacional e o desmatamento generalizado continuam sendo fatores importantes. As alterações ambientais também afetam a epidemiologia das leishmanioses, tendo relação mais direta com fatores como mudanças na urbanização e pela incursão humana em áreas de floresta. Mudanças de temperatura, precipitação e umidade podem ter fortes efeitos sobre vetores e hospedeiros de reservatórios, alterando sua distribuição e influenciando sua sobrevivência e tamanho da população. Assim, o impacto no ciclo desses parasitos 
pode contribuir para a transmissão da doença em áreas anteriormente não endêmicas (WHO, 2020).

Embora estime-se que ocorram anualmente 700.000 a 1 milhão de novos casos e cerca de 26.000 a 65.000 mortes, ainda há uma disponibilidade de recursos muito aquém da necessária para que as leishmanioses sejam erradicadas (WHO, 2020), pois despertam pouco interesse por parte das grandes empresas farmacêuticas para investimento em pesquisa, produção de medicamentos mais eficazes e vacinas (MURRAY et al., 2005; LINDOSO; LINDOSO, 2009; FEASEY et al., 2010).

De acordo com a Organização Mundial de Saúde (WHO, 2020), em 2017, 20.792 dos 22.145 novos casos relatados à OMS ocorreram em sete países: Brasil, Etiópia, Índia, Quênia, Somália, Sudão do Sul e Sudão. Ou seja, 94\% dos casos de leishmaniose ao redor do mundo estão concentrados em apenas sete países. Ainda há uma enorme dificuldade na obtenção de dados epidemiológicos. Assim como outras doenças tropicais negligenciadas, as leishmanioses não são de notificação compulsória em todos os países em que ocorrem e muitos deles não realizam vigilância ou outras investigações, além de não possuírem um sistema de armazenamento de dados. A subnotificação é um fato que merece destaque pelas autoridades de saúde, pois são necessários dados que reflitam de maneira mais fidedigna a realidade acerca da doença para que medidas de controle mais efetivas possam ser tomadas.

As características clínicas das leishmanioses são de grande diversidade, variando conforme um conjunto de fatores que envolvem a virulência e genética do parasito, a espécie do flebótomo, fatores ambientais, bem como a genética e capacidade imunológica do hospedeiro mamífero (MURRAY et al., 2005). De acordo com as manifestações clínicas, essas doenças são divididas em dois grupos: leishmaniose visceral (LV) e leishmaniose tegumentar (LT). A LT se subdivide em quatro tipos básicos: leishmaniose cutânea localizada, disseminada, cutânea difusa e mucocutânea (DAVID; CRAFT, 2009; GOTO; LINDOSO, 2012).

A leishmaniose visceral é uma forma grave da doença, que recebe esse nome por acometer as vísceras, causando organomegalia, especialmente do fígado e baço. É popularmente conhecida como calazar e caracteriza-se por episódios 
irregulares de febre alta, considerável perda de peso, anemia, pancitopenia e discreto aumento da pigmentação da pele em alguns pacientes. Os agentes etiológicos relacionados a essa forma clínica são Leishmania donovani e $L$. infantum/chagasi. A leishmaniose dérmica pós-calazar (PKDL) é uma complicação da leishmaniose visceral que pode aparecer entre seis meses a um ano ou mais após cura aparente da doença, embora possa ocorrer antes ou mesmo simultaneamente com a LV. A PKDL é caracterizada por máculas hipopigmentadas, pápulas, erupção cutânea e/ou nodular e ocorre principalmente em áreas onde $L$. donovani é endêmica (GONZÁLEZ et al., 2017).

A leishmaniose cutânea localizada é considerada a forma mais comum da doença, podendo ter início como pequenas pápulas vermelhas e progredir para úlceras características com bordas elevadas, de centro granuloso e avermelhado, nas regiões de picada do inseto vetor ou em áreas próximas. As úlceras são confinadas à derme e geralmente acometem partes expostas do corpo, como a face, braços e pernas (DASSONI et al., 2013; TORRES- GUERRERO et al., 2017). Essas lesões tendem à cicatrização espontânea e normalmente o período de cura é de alguns meses, ainda que não haja intervenção medicamentosa. A forma cutânea localizada da leishmaniose é provocada por parasitos pertencentes tanto ao subgênero Leishmania como Viannia. No Brasil, ela é causada principalmente pela espécie L. (V.) braziliensis. (GOTO; LINDOSO, 2012).

A leishmaniose do tipo disseminada é caracterizada por múltiplas lesões em diversas áreas do corpo, que são encontradas em locais distantes de onde ocorreram as picadas pelo inseto vetor, provavelmente devido à disseminação hematológica ou linfática do parasito (REITHINGER et al., 2007; TORRES-GUERRERO et al., 2017). Podem ser ulceradas, papulosas, nodulares e acneiformes, e normalmente possuem boa resposta clínica à terapia medicamentosa. As espécies envolvidas nessa forma clínica são a L. (V.) braziliensis, L. (V.) guyanensis, e L. (L) amazonensis, sendo a primeira a mais comumente associada a esta forma clínica (DAVID; CRAFT, 2009; GOTO; LINDOSO, 2012).

A leishmaniose cutânea difusa é uma manifestação mais grave e rara da LT. É caracterizada por numerosas lesões cutâneas não ulcerosas, nódulos e placas densamente infiltradas com parasitos, que podem disseminar do sítio de infecção 
para todo o corpo por via hematogênica ou linfática (REITHINGER et al., 2007; TORRES-GUERRERO et al., 2017). Indivíduos imunocomprometidos estão mais propensos a desenvolver esse tipo de leishmaniose e a principal espécie de Leishmania relacionada é L. (L.) amazonensis (DAVID; CRAFT, 2009; GOTO; LINDOSO, 2012).

Por fim, a forma mucocutânea é caracterizada pelo seu início como uma inflamação da mucosa nasal, com evidente obstrução da região, progredindo para perfuração de septo e destruição da cartilagem nasal. Pode haver acometimento secundário de lábios, palato mole, faringe, laringe e bochechas, causando desfiguração dessas áreas (DAVID; CRAFT, 2009). A principal característica da LMC é o dano às mucosas e cartilagens. Essa forma clínica normalmente não responde bem aos tratamentos disponíveis e, por isso, são relatados altos índices de reicidivas. Os agentes etiológicos mais associados a este tipo de infecção são $L$. (V.) braziliensis e L. (V.) guananensis (TORRES-GUERRERO et al., 2017).

São descritas 22 espécies de Leishmania patogênicas ao homem. Esses parasitos possuem um ciclo de vida heteroxênico, alternando-se entre um hospedeiro invertebrado e um vertebrado (FRAGA et al., 2010; BATES, 2018). Os hospedeiros invertebrados são dípteros da família Psychodidae, subfamília Phlebotominae. Os vetores da leishmaniose para os hospedeiros vertebrados são as fêmeas desses insetos, sendo as espécies pertencentes ao gênero Lutzomia mais prevalentes no Novo Mundo e as do gênero Phlebotomus, no Velho Mundo (KAMHAWI, 2006).

O ciclo biológico do parasito encontra-se exemplificado na figura 1. A transmissão ocorre no momento do repasto sanguíneo, onde o flebótomo infectado regurgita formas promastigotas infecctantes no momento em que se alimenta do sangue do hospedeiro humano. As formas promastigotas são alongadas, flageladas e móveis. Quando no interior do humano, as promastigotas são fagocitadas por células do sistema de defesa, principalmente macrófagos. No interior dessas células, as promastigotas sofrem uma série de alterações que lhes conferem uma nova forma, denominada amastigota. As amastigotas são intracelulares, imóveis e não possuem flagelo exteriorizado. Embora o ambiente seja muito ácido, isso favorece sua multiplicação por divisão binária no interior dos macrófagos, pois os parasitos 
apresentam estratégias sofisticadas que fazem com que eles sejam capazes de sobreviver nessas condições adversas, driblando as ações microbicidas do sistema imunológico hospedeiro (CHARMOY et al., 2010; BATES, 2018).

Com a multiplicação, a célula hospedeira é lisada e libera para o meio extracelular as formas amastigotas que continha em seu interior. Essas amastigotas conseguem infectar novas células, dando continuidade ao processo infeccioso. Além disso, o rompimento celular promove reações inflamatórias que, juntamente com outros fatores, desencadeiam padrões de resposta imunológica característicos de cada tipo clínico de leishmaniose. Quando o inseto vetor se alimenta do sangue do vertebrado infectado, ele ingere também formas amastigotas que estão presentes no local. Estas, ao chegarem-no intestino médio ou estômago do vetor, sofrem um primeiro ciclo replicativo, transformando-se em formas extracelulares flageladas denominadas promastigotas procíclicas (KAMHAWI, 2006; BATES, 2018).

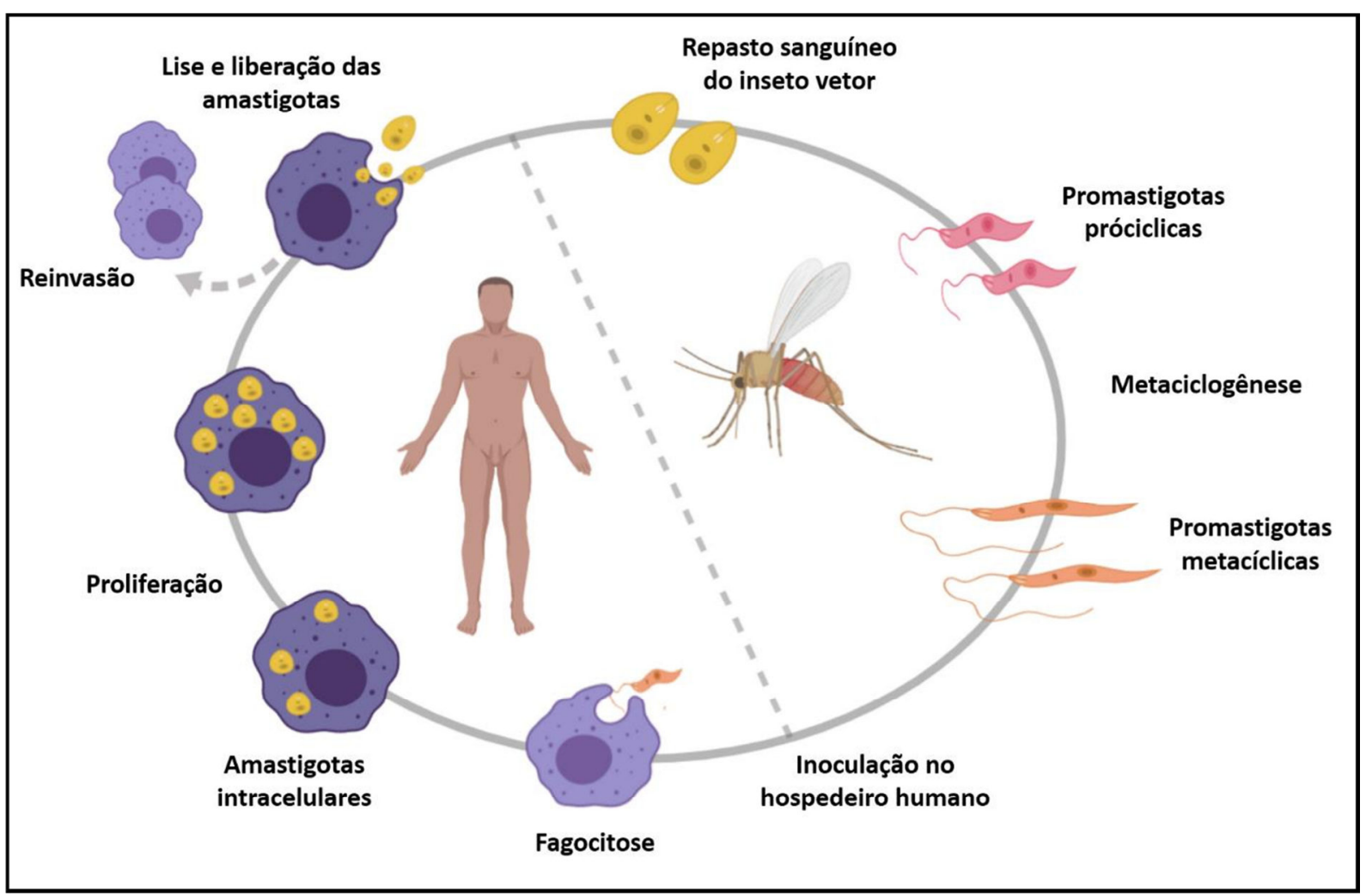

Figura 1 - Ciclo biológico de Leishmania spp.

Fonte: Elaboração própria, 2020. 
As promastigotas procíclicas, entretanto, ainda não são a forma infectante de Leishmania spp. Para tal, elas precisam passar pelo processo de metaciclogênese, que ocorre no interior do inseto vetor. A mataciclogênese promove alterações na expressão gênica dos parasitos e leva a um aumento a infectividade e mobilidade. As formas procíclicas possuem corpo celular largo e arredondado e um flagelo pequeno, enquanto que as metacíclicas apresentam corpo celular mais fino e um flagelo com aproximadamente o dobro do tamanho do seu corpo celular. O corpo mais fino e o flagelo bem desenvolvido fazem com que o parasito tenha uma maior capacidade de locomoção e assim consiga sobreviver durante o processo de infecção no hospedeiro vertebrado (Figura 2). As promastigotas metacíclicas migram para a porção bucal do inseto vetor e no momento em que é realizado um novo repasto sanguíneo elas podem ser inoculadas no hospedeiro vertebrado, iniciando um novo ciclo de infecção (DA SILVA et al., 2015; BATES, 2018).

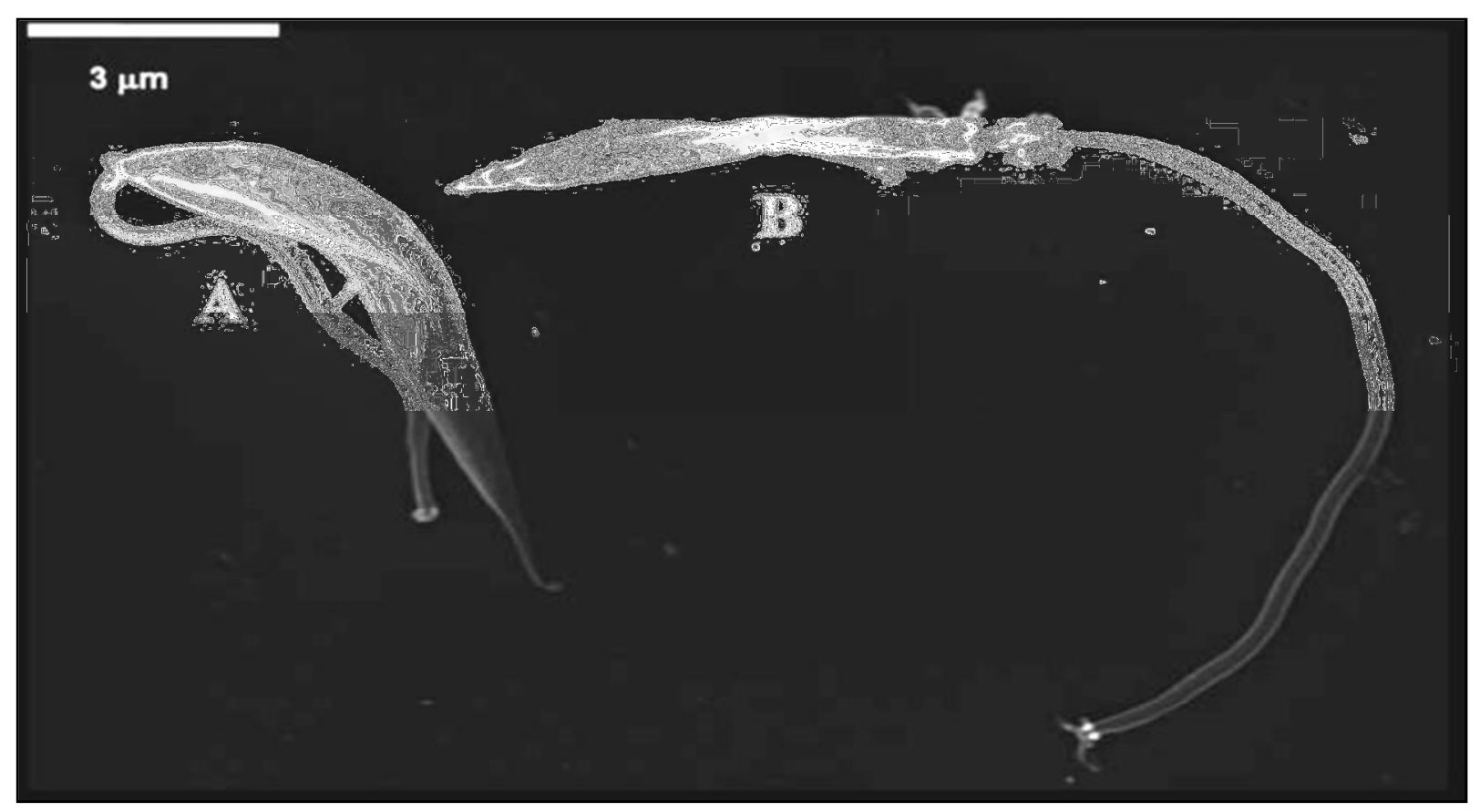

Figura 2 - Forma promastigota procíclica e metacíclica de Leishmania sp.

Fonte: SOARES et al., 2005. Fotomicrografia obtida por análise através de microscopia eletrônica de varredura. (A) Forma promastigota procíclica e (B) metacíclica de Leishmania sp. 
Além das alterações no corpo celular e tamanho do flagelo, a metaciclogênese leva a modificações de moléculas presentes na superfície do parasito e também na expressão de enzimas, de modo que aumente a capacidade do protozoário em escapar dos mecanismos de defesa do hospedeiro e também aumente seu potencial de invasão tecidual para infecção. Assim, a metaciclogênese está relacionada a um aumento na expressão de diversos fatores de virulência de Leishmania spp. (ATAN et al., 2018; MENDES et al., 2019). Ainda nesse contexto, o termo "fitness" é utilizado para referir-se ao conjunto de fatores que garantem a um patógeno a capacidade crescer, sobreviver, reproduzir-se e transmitir doença. A virulência está, muitas vezes, associada ao fitness, pois refere-se ao potencial de um patógeno em causar dano no hospedeiro (KAUR; RAJPUT, 2014).

Na prática clínica, devido à ausência de vacinas disponíveis para uso clínico e a dificuldade em controlar os vetores e reservatórios para a doença, a principal ferramenta para o combate da leishmaniose ainda é a farmacoterapia. Contudo, o arsenal terapêutico é bastante limitado e consiste nos antimoniais pentavalentes, anfotericina B (e suas formulações lipídicas), pentamidina, miltefosina e paromomicina. Na maioria dos países, como no Brasil, as drogas de primeira escolha para o tratamento das infecções por Leishmania sp. são os antimoniais pentavalentes (SbV): Estibogluconato de sódio e antimoniato de meglumina. Entretanto, os índices de resistência a estas drogas vem crescendo com o passar dos anos. Em casos de insucesso terapêutico, opta-se principalmente pelo tratamento com anfotericina B e pentaminidina, podendo ser feito uso isolado ou em associações, mas o alto custo do tratamento e os intensos efeitos colaterais limitam o uso destas drogas (ALVAR; CROFT; OLLIARO, 2006; ULIANA et al., 2018).

Alguns estudos em procariotos e eucariotos demonstram que a resistência a drogas está relacionada a alterações no fitness dos micro-organismos, onde é possível observar modificações no crescimento, virulência e transmissibilidade entre hospedeiros (COHEN et al., 2003; ZHANG et al., 2006; ROSENTHAL, 2013; DA SILVA; DOMINGUES, 2017). Visto que tais alterações podem influenciar diretamente no curso da infecção, alterando o prognóstico clínico, é relevante que essa 
associação entre resistência a drogas e virulência/fitness parasitário seja melhor estudada.

\section{OBJETIVO}

Este trabalho tem como objetivo avaliar, através de revisão bibliográfica, se a resistência aos antimoniais está correlacionada com o aumento da virulência de parasitos do gênero Leishmania.

\section{METODOLOGIA}

Trata-se de um estudo descritivo, realizado a partir das pesquisas bibliográficas e documentais para subsidiar a discussão do tema. A pesquisa foi realizada entre os meses de dezembro a fevereiro de 2020.

Como critérios de inclusão, foram inseridos artigos científicos e documentos de órgãos oficiais publicados nas últimas duas décadas (2000-2020) que se encontram dentro do escopo do presente trabalho e possuem texto integral disponibilizado para leitura.

A busca foi realizada utilizando as bases de dados Scielo, Google Acadêmico, PubMed, Periódicos CAPES e Elsevier utilizando as palavras-chaves em português: Leishmania spp.; Antimoniais; Virulência; Resistência; Fitness parasitário. Também foi realizada a busca através das respectivas traduções para o idioma inglês: Leishmania spp.; Antimonials; Virulence; Resistance; Parasite fitness. Foram feitas diferentes combinações dos descritores para extensiva análise dos dados disponíveis na literatura. 


\section{RESULTADOS E DISCUSSÃO}

Os antimoniais pentavalentes (SbV) são mais tolerados e considerados mais seguros que os trivalentes, seus percussores. Ainda assim, vários efeitos adversos são comumente relatados, tais como: dores abdominais, náuseas, vômitos, artralgia, letargia, convulsões, pancreatite, cardiotoxicidade e nefrotoxicidade. A administração destes medicamentos deve ser feita por via intramuscular ou intravenosa, com infusão lenta para que seja evitada a ocorrência de trombose. O paciente deve ser monitorado, realizando periodicamente eletrocardiogramas, hemogramas e dosagens séricas, pois o uso de SbV pode causar alterações cardíacas, leucopenia, anemia, trombocitopenia e elevação dos níveis das enzimas hepáticas (ZAGHLOUL; ALJASSER, 2004; SHAHIAN; ALBORZI, 2009).

Além dos efeitos tóxicos associados ao tratamento com os antimoniais, longos períodos de terapia e insucessos terapêuticos são também observados. O esquema de tratamento com antimoniais pentavalentes duram, em média, 20 a 30 dias, mas tem sido modificado ao longo dos anos quanto à dose e duração, visando minimizar o aparecimento de parasitos resistentes. Entretanto, casos de resistência aos SbV têm sido constatados, trazendo um novo desafio para o tratamento da doença. Acredita-se que o surgimento de cepas resistentes seja influenciado pela espécie de Leishmania envolvida, por variações farmacocinéticas do indivíduo e variações de interação da droga com o sistema imune do hospedeiro (THAKUR et al., 2001; ASHUTOSH et al., 2007).

Alguns estudos em procariotos e eucariotos demonstram que a resistência a drogas está normalmente relacionada a uma redução no fitness dos microorganismos, pois observa-se decréscimo no crescimento, virulência e transmissibilidade entre hospedeiros (BABIKER; HASTINGS; SJÖBERG, 2009; ABDELRAOUF et al., 2011). Entretanto, pesquisas realizadas na última década têm demonstrado que algumas espécies de Leishmania, principalmente L. donovani, resistentes a drogas parecem ter desenvolvido mutações compensatórias que permitiram ao protozoário restaurar, total ou parcialmente, as habilidades de fitness. 
Em Leishmania sp. essa habilidade tem sido relacionada à capacidade de gerar formas metacíclicas, que possuem maior poder de infecção no hospedeiro (OUAKAD et al., 2011; VANAERSCHOT et al., 2013). A natureza plástica do genoma desses parasitos faz com que eles sejam altamente adaptáveis, sendo capazes de modular a expressão gênica através da amplificação ou deleção de genes e alterar a ploidia cromossomal em resposta ao estresse (HENDRICKX et al., 2016).

Vanaerschot e colaboradores (2010) analisaram isolados clínicos de $L$. donovani suscetíveis e resistentes antimonial e observaram que parasitas resistentes apresentaram menor sensibilidade ao estresse oxidativo e nitrosativo in vitro. A taxa de infecção foi significativamente maior nos grupos infectados por cepas resistentes à droga. Tais cepas também foram mais infecciosas in vivo, atingindo uma maior carga parasitária no baço e no fígado de camundongos Balb/c infectados. Os autores sugerem que maior tolerância ao estresse e maior número de formas metacíclicas podem contribuir para que cepas resistentes tenham habilidades de sobrevivência superior, tanto nas formas promastigotas como nas amastigotas, quando comparadas às cepas sensíveis.

Ouakad et al. (2011) demonstraram, em estudos utilizando L. donovani, que as cepas resistentes aos antimoniais apresentaram números duas vezes maior de formas metacíclicas do que os parasitos sensíveis à droga. Além disso, as culturas resistentes também apresentaram maior capacidade de infecção em macrófagos murinos. Outro fator adicional observado pelos autores foi a maior resistência dessas cepas à lise mediada pelo sistema complemento, que é o primeiro mecanismo de defesa do sistema imunológico à entrada dos parasitos no organismo.

O sistema complemento exerce um importante papel durante a infecção por Leishmania spp. No momento em que o inseto vetor insere as formas promastigotas no hospedeiro mamífero elas entram em contato com o plasma. Uma reação extremamente rápida do sistema complemento é então desencadeada, principalmente por meio da via alternativa e da via clássica, conseguindo eliminar grande parte da população de parasitos. Acredita-se que a ação do sistema complemento na fase inicial da infecção seja um dos fatores responsáveis por determinar se a infecção será controlada ou estabelecida (ROSSI; FASEL, 2018). 
Contudo, a eficácia do sistema complemento está diretamente relacionada com a fase de desenvolvimento dos protozoários no momento da inoculação no hospedeiro mamífero. As formas promastigotas metacíclicas passam por alterações morfológicas e bioquímicas que as tornam mais resistentes à lise mediada pelo sistema complemento em comparação com as formas procíclicas (LAURENTI et al., 2004). Estudos comprovam que as culturas de promastigotas em fase estacionária de crescimento, que possuem majoritariamente formas metacíclicas, são mais resistentes à lise mediada pelo sistema complemento quando comparadas com culturas em fase logarítmica (formas procíclicas) (PINTO-DA- SILVA et al., 2002; Da SILVA et al., 2015).

Assim, o achado de Ouakad et al. (2011) sugere que parasitos resistentes aos antimoniais são capazes de atingir maiores índices de formas metacíclicas, as quais são mais resistentes à ação do sistema complemento e isso pode ser um dos fatores que contribui para a maior capacidade de infecção em macrófagos murinos que foi observada pelos autores. Caso esse comportamento seja observado também em outras espécies de Leishmania e em outros hospedeiros através de estudos in vivo, pode demonstrar uma correlação direta entre a resistência aos antimoniais e a maior virulência dos parasitos que, consequentemente, altera o curso da infecção.

Vanaerschot et al. (2011) também analisaram cepas de L. donovani resistentes aos antimoniais e identificaram maior capacidade de infecção in vivo quando comparadas aos respectivos parasitos sensíveis ao fármaco. Outra pesquisa foi realizada por Moura et al. (2016) com L. infantum isoladas de pacientes refratários ao tratamento com antimonial (resistentes ao fármaco) e outro grupo, controle, com cepas isoladas de pacientes responsivos à droga. Os autores verificaram que o índice de infecção em macrófagos foi maior naqueles infectados com as cepas isoladas de pacientes refratários ao tratamento em comparação ao grupo controle e correlacionaram esse achado com a produção aumentada de interleucina 1- $\beta$, além disso, esses parasitas estimulavam citocinas inflamatórias e eram resistentes aos mecanismos de eliminação de macrófagos, sendo estes fatores que podem contribuir para gravidade da doença. 


\section{CONCLUSÃO}

Diante das poucas opções terapêuticas existentes para o tratamento das leishmanioses, a elevada toxicidade dos fármacos e o alto índice de resistência dos parasitos aos medicamentos, os achados sobre a possível correlação entre resistência aos antimoniais e aumento virulência em protozoários do gênero Leishmania levantam uma questão fundamental sobre os riscos potenciais de selecionar mais patógenos virulentos através de intervenções maciças de quimioterapia. Tais estudos ainda são escassamente encontrados na literatura, mas apontam a necessidade dessa correlação ser melhor estudada, de modo a esclarecer as alterações moleculares envolvidas nesses processos e poder otimizar a farmacoterapia dessas infecções, impactando diretamente na prática clínica.

\section{REFERÊNCIAS BIBLIOGRÁFICAS}

ABDELRAOUF, K.; KABBARA, S.; LEDESMA, K. R.; POOLE, K.; TAM, V. H. Effect of multidrug resistance-conferring mutations on the fitness and virulence of Pseudomonas aeruginosa. Journal of Antimicrobial Chemotherapy, v. 66, pp. 1311-17, 2011.

ATAN, N. A. D.; KOUSHKI, M.; AHMADI, N. A.; REZAEI-TAVIRANI, M. Metabolomics-based studies in the field of Leishmania/leishmaniasis. Alexandria journal of medicine, v. 54, n. 4, p. 383-390, 2018.

ASHUTOSH; SUNDAR, S.; GOYAL, N. Molecular mechanisms of antimony resistance in Leishmania. Journal of Medical Microbiology, v. 56, pp. 143-53, 2007.

BABIKER, H. A.; HASTINGS, I. M.; SWEDBERG, G. Impaired fitness of drug-resistant malaria parasites: evidence and implication on drug-deployment policies. Expert Review of Antiinfective Therapy, v. 7, pp. 581-93, 2009.

BATES, P. A. Revising Leishmania's life cycle. Nature microbiology, v. 3, n. 5, p. 529-530, 2018.

CHARMOY, M.; AUDERSET, F.; ALLEBACH, C.; TACCHINI-COTTIER, F. The prominent role of neutrophils during the initial phase of infection by Leishmania parasites. Journal of Biomedicine and Biotechnology, v. 2010, pp. 1-8, 2010.

COHEN, T.; SOMMERS, B.; MURRAY, M. The effect of drug resistance on the fitness of Mycobacterium tuberculosis. The Lancet infectious diseases, v. 3, n. 1, p. 13-21, 2003.

DA SILVA, I. A.; MORATO, C. I.; QUIXABEIRA, V. B. L.; PEREIRA, L. I. A.; DORTA, M. L.; 
OLIVEIRA, M. A. P.; HORTA, M. F.; RIBEIRO-DIAS, F. In vitro metacyclogenesis of Leishmania (Viannia) braziliensis and Leishmania (Leishmania) amazonensis Clinical Field Isolates, as Evaluated by Morphology, Complement Resistance, and Infectivity to Human Macrophages. BioMed Research International, v. 2015, 2015.

DA SILVA, G. J.; DOMINGUES, S. Interplay between colistin resistance, virulence and fitness in Acinetobacter baumannii. Antibiotics, v. 6, n. 4, p. 28, 2017.

DASSONI, F.; ABEBE, Z.; NAAFS, B.; MORRONE, A. Cutaneous and mucocutaneous leishmaniasis resembling borderline-tuberculoid leprosy: a new clinical presentation? Acta dermato-venereologica, v. 93, n. 1, pp. 74-77, 2013.

DAVID, C.V. CRAFT, N. Cutaneous and mucocutaneous leishmaniasis. Dermatologic Therepy, v. 22. pp. 491-502, 2009.

FEASEY, N.; WANSBROUGH-JONES, M.; MABEY, D. C. W.; SOLOMON, A. W. Neglected tropical diseases. British Medical Bulletin, v. 93, pp. 179-200, 2010.

FRAGA, J.; MONTALVO, A. M.; DONCKER, S. D.; DUJARDIN, J. C.; AUWERA, G. V. A. Phylogeny of Leishmania species based on the heat-shock protein 70 gene. Infection, Genetics and Evolution, v. 10, pp. 238-245, 2010.

GONZÁLEZ, U; PINART, M.; KALITA, S.; HERRERO, M.; VÉLEZ, I.D.; SUNDAR, S.; ALVAR, J. Interventions for visceral leishmaniasis. The Cochrane database of systematic reviews, $v$. 2017, n. 8, 2017.

GOTO, H.; LINDOSO, J. A. L. Cutaneous and mucocutaneous leishmaniasis. Infectious Disease Clinics of North America, v. 26, pp. 293-307, 2012.

HENDRICKX, S.; GUERIN, P. J.; CALJON, G.; CROFT, S. L.; MAES, L. Evaluating drug resistance in visceral leishmaniasis: the challenges. Parasitology, pp. 1-11, 2016.

KAMHAWI, S. Phlebotomine sand flies and Leishmania parasites: friends or foes? Trends in Parasitology, v. 22, n. 9, pp. 439-445, 2006.

KAUR, G.; RAJPUT, B. Comparative analysis of the omics technologies used to study antimonial, amphotericin $\mathrm{B}$, and pentamidine resistance in Leishmania. Journal of parasitology research, v. 2014, 2014.

LAURENTI, M. D.; ORN, A.; SINHORINI, I. L.; CORBETT, C. E. P. The role of complement in the early phase of Leishmania (Leishmania) amazonensis infection in BALB/c mice. Brazilian Journal of Medical and Biological Research, v. 37, pp. 427-34, 2004.

LINDOSO, J. A. L.; LINDOSO, A. A. B. P. Neglected Tropical Diseases in Brazil. Revista do Instituto de Medicina Tropical de São Paulo, v. 51, n. 5, pp. 247-253, 2009.

MENDES, B. P.; DA SILVA, I. A.; DAMATA, J. P.; CASTRO-GOMES, T.; VIEIRA, L. Q.; RIBEIRO-DIAS, F.; HORTA, M. F. Metacyclogenesis of Leishmania (Viannia) guyanensis: a comprehensive study of the main transformation features in axenic culture and purification of metacyclic promastigotes by negative selection with Bauhinia purpurea lectin. Parasitology, v. 146, n. 6, p. 716-727, 2019.

MOURA, T. R.; SANTOS, M. L.; BRAZ, J. M.; SANTOS, L. F.; ARAGÃO, M. T.; OLIVEIRA, F. A.; SANTOS, P. L.; SILVA, A. M.; JESUS, A. R.; ALMEIDA, R. P. Crossresistance of Leishmania infantum isolates to nitric oxide from patients refractory to antimony treatment, and greater tolerance to antileishmanial responses by macrophages. Parasitology Research. v. 115, n. 2, pp. 713-21, 2016. 
MURRAY, H. W.; BERMAN, J. D.; DAVIES, C. R.; SARAVIA, N. G. Advances in leishmaniasis. Lancet, v. 366, pp. 1561-1577, 2005.

OUAKAD, M.; VANAERSCHOT, M.; RIJAL, S.; SUNDAR, S.; SPEYBROECK, N.; KESTENS, L.; BOEL, L.; DONCKER, D.; MAES, I.; DECUYPERE, S.; DUJARDIN, J. C. Increased metacyclogenesis of antimony-resistant Leishmania donovani clinical lines. Parasitology, $\mathrm{v}$. 138, n. 11, pp. 1392-99, 2011.

PINTO-DA-SILVA, L. H.; FAMPA, P.; SOARES, D. C.; OLIVEIRA, S. M. P.; SOUTOPADRON, T.; SARAIVA, E. M. The 3A1-La monoclonal antibody reveals key features of Leishmania (L.) amazonensis metacyclic promastigotes and inhibits procyclics attachment to the sand fly midgut. International Journal for Parasitology, v. 35, n. 7, pp. 757-64, 2005.

REITHINGER, R.; DUJARDIN, J. C.; LOUZIR, H.; PIRMEZ, C.; ALEXANDER, B.; BROOKER, S. Cutaneous leishmaniasis. The Lancet Infectious Diseases, v. 7, n. 9, pp. 581- 96, 2007. ROSENTHAL, P. J. The interplay between drug resistance and fitness in malaria parasites. Molecular microbiology, v. 89, n. 6, p. 1025-1038, 2013.

ROSSI, M.; FASEL, N.. How to master the host immune system? Leishmania parasites have the solutions!. International immunology, v. 30, n. 3, p. 103-111, 2018.

SHAHIAN, M.; ALBORZI, A. Effect of meglumine antimoniate on the pancreas during treatment of visceral leishmaniasis in children. Medical Science Monitor, v. 15, 2009.

SJÖBERG, A. P.; TROUW, L. A.; BLOM, A. M. Complement activation and inhibition: a delicate balance. Trends in Immunology, v. 30, n. 2, pp. 83-90, 2009.

SOARES, R. P. P.; CARDOSO, T. L.; BARRON, T.; ARAÚJO, M. S. S.; PIMENTA, P. F. P.; TURCO, S. J. Leishmania braziliensis: a novel mechanism in the lipophosphoglycan regulation regulation during metacyclogenesis. International Journal for Parasitology, v. 35, pp. 245253, 2005.

THAKUR, C. P.; DEDET, J. P.; NARAIN, S.; PRATLONG, F. Leishmania species, drug unresponsiveness and visceral leishmaniasis in Bihar, India. Transactions of the Royal Society of Tropical Medicine and Hygiene. v. 95, n. 2, pp. 187-189, 2001.

TORRES-GUERRERO, E.; QUINTANILLA-CEDILLO, M.R.; RUIZ-ESMENJAUD, J.; ARENAS, R. Leishmaniasis: a review. F1000Research, v. 6, 2017.

ULIANA, S. R. B; TRINCONI, C. T.; COELHO, A. C. Chemotherapy of leishmaniasis: present challenges. Parasitology, v. 145, n. 4, p. 464-480, 2018.

VANAERSCHOT, M.; MAES, I.; OUAKAD, M.; ADAUI, V.; MAES, L.; DONCKER, S.; RIJAL, S.; CHAPPUIS, F.; DUJARDIN, J.; DECUYPERE, S. Linking in vitro and in vivo survival of clinical Leishmania donovani strains. Plos one. v. 5, n. 8, pp. e12211, 2010.

VANAERSCHOT, M.; DONCKER, S.; RIJAL, S.; MAES, L.; DUJARDIN, J.; DECUYPERE, S. Antimonial resistance in Leishmania donovani is associated with increased in vivo parasite burden. Plos one, v. 6, n. 8, pp. e23120, 2011.

VANAERSCHOT, M.; DECUYPERE, S.; BERG, M.; ROY, S.; DUJARDIN, J. C. Drug resistant microorganisms with a higher fitness-can medicines boost pathogens?. Critical reviews in microbiology, v. 39, n. 4, pp. 384-94, 2013.

WORLD HEALTH ORGANIZATION. Media centre. Leishmaniasis. 2020. Disponível em: < http://www.who.int/mediacentre/factsheets/fs375/en/> Acesso em: 20/02/2010.

ZAGHLOUL, I. Y.; AL-JASSER, M. Effect of renal impairment on the pharmacokinetics of 
antimony in hamsters. Annals of Tropical Medicine and Parasitology, v. 98, pp. 793-800, 2004.

ZERPA O, ULRICH M, BLANCO B, POLEGRE M, AVILA A, MATOS N, MENDOZA I, PRATLONG F, RAVEL C, CONVIT J. Diffuse cutaneous leishmaniasis responds to miltefosine but then relapses. Britanic Journal of Dermatology, v. 156, pp. 1328-1335, 2007.

ZHANG, Q.; SAHIN, O.; MCDERMOTT, P. F.; PAYOT, S. Fitness of antimicrobial-resistant Campylobacter and Salmonella. Microbes and Infection, v. 8, n. 7, p. 1972-1978, 2006. 